\title{
Clinical Outcomes of Isolated Regional Lymph Node Recurrence in Patients With Malignant Cutaneous Melanoma
}

\author{
JEANNY KWON ${ }^{*}$, YOUNG A. KIM ${ }^{2}$, CHEOL LEE ${ }^{3}$, BYOUNG HYUCK KIM ${ }^{4,5}$, SUZY KIM $^{4}$ and IL HAN KIM ${ }^{5,6}$ \\ ${ }^{1}$ Department of Radiation Oncology, Chungnam National University Hospital, Daejeon, Republic of Korea; \\ ${ }^{2}$ Department of Pathology, Seoul Metropolitan Government Seoul National \\ University Boramae Medical Center, Seoul, Republic of Korea; \\ ${ }^{3}$ Department of Pathology, Seoul National University College of Medicine, Seoul, Republic of Korea; \\ ${ }^{4}$ Department of Radiation Oncology, Seoul Metropolitan Government Seoul \\ National University Boramae Medical Center, Seoul, Republic of Korea; \\ ${ }^{5}$ Department of Radiation Oncology, Seoul National University College of Medicine, Seoul, Republic of Korea; \\ ${ }^{6}$ Cancer Research Institute, Seoul National University, Seoul, Republic of Korea
}

\begin{abstract}
Background/Aim: Regional lymph node recurrence $(R L N R)$ is the most common pattern of recurrence within 2 years from the diagnosis of patients with non-metastatic malignant cutaneous melanoma. However, isolated RLNR without distant metastasis has been rarely studied. Patients and Methods: Forty patients with isolated RLNR as a first recurrence were analyzed retrospectively. The clinical outcomes and prognostic impact of clinicopathologic parameters were analyzed. Immunostaining for FOXP3, $V E G F, p A K T$, and pS6 was also performed. Results: The median disease-free interval from first diagnosis to isolated $R L N R$ and post-recurrence recurrence-free survival (pRFS) were 12 months and 7.2 months, respectively. Distant failure was the most common pattern of failure after isolated RLNR (67.5\%). The number of initially harvested lymph nodes $(L N)$ $>7$ and $L N$ ratio $>22.2 \%$ at the time of recurrence were prognosticators for $p R F S$ in multivariate analysis. None of the tested biomarkers were significantly related to prognosis. The 5-year post-recurrence overall survival rate was $84.9 \%$. Conclusion: Most patients with isolated RLNR will experience a second failure within months, especially distantly. The number of initially harvested $L N S$ and $L N$ ratio at the time of recurrence could predict $p R F S$.
\end{abstract}

*These Authors contributed equally to this work.

Correspondence to: Suzy Kim, MD, Ph.D., Department of Radiation Oncology, Seoul Metropolitan Government Seoul National University Boramae Medical Center, 20 Boramae-ro 5-gil, Dongjak-gu, Seoul 07061, Republic of Korea. Tel: +82 28701691, Fax: +82 28701689, e-mail: suzy101@snu.ac.kr

Key Words: Melanoma, lymph node, recurrence, lymph node ratio.
Malignant melanoma is the fifth most common cancer in men and the sixth in women in the United States. It has also been reported that the incidence is increasing in all age groups and tumor thickness categories. Early-stage malignant melanoma is usually cured by radical surgery with a 5-year survival rate of $99 \%$. However, the survival rate decreases to $63 \%$ when the melanoma is accompanied by regional lymph node (LN) metastasis (1). Considering the clinical significance of LN metastasis and its $15-20 \%$ incidence in malignant melanoma patients, surgical LN evaluation should be considered from a relatively early stage $(2,3)$. Immediate lymphadenectomy using sentinel LN biopsy significantly increased survival in patients with nodal metastasis (2).

The most common failure site of resected malignant melanoma, especially within the first two years after primary excision, has been the regional $\mathrm{LN}$ area $(4,5)$. Because of the dismal prognosis with a median survival of 5-14 months and a 5-year survival rate of $8-33 \%$ after regional LN recurrence (RLNR), there have been many studies focused on predicting LN recurrence (4-6). Through these studies, important factors associated with RLNR were identified such as the location of the nodal basin, number of metastatic LNs (NMLN), size of metastatic LNs, presence of extracapsular extension, patient age, etc. Based on these findings, treatment strategies such as adjuvant radiotherapy (RT) have been tried in patients who are at high risk for $\operatorname{RLNR}(7,8)$.

However, there have been few studies on the course of disease, prognostic factors, and treatment strategies for the RLNR of malignant melanoma. In particular, isolated RLNR without any evidence of distant metastasis or local recurrence is rare, comprising about $10 \%$ of the patients with RLNR (6). Although many salvage strategies for isolated RLNR have been attempted based on the advancements of numerous treatment techniques for various solid tumors (9, 
10), the clinical data is scant for isolated RLNR in malignant melanoma due to its rarity. If the RLNR occurred without distant metastasis, despite the general tendency of distant metastasis of malignant melanoma, it would suggest a relatively low systemic tumor burden. A strategy to improve the locoregional control rate in such cases will be important in the same context as considering adjuvant RT for patients with a high risk of RLNR after initial surgery.

Thus, we aimed to explore the disease course and prognostic factors, including not only clinical factors but also biomarkers, to find out if there are any subgroups that need different treatment strategies after isolated RLNR.

\section{Patients and Methods}

Patient selection. To identify the patients who experienced isolated RLNR after obtaining disease-free status for cutaneous malignant melanoma, we performed the following search. Using the in-hospital electronic database, we searched for patients with the word "melanoma" on their chart, "recurrence" or "recurrent" in the imaging study results, and "melanoma" reported on the pathologic reports. Through this process, we conducted a retrospective medical chart review of all patients who were diagnosed with malignant cutaneous melanoma pathologically and had underwent an imaging study for recurrence surveillance at Seoul National University Hospital and Seoul Metropolitan Government-Seoul National University Boramae Medical Center. The inclusion period was from January 2000 to October 2014. The patients who had distant metastasis at initial diagnosis, those with simultaneous distant or local recurrence at the time of RLNR, or those who received palliative or supportive treatment only for recurrent disease were excluded. Finally, we included 40 patients who had experienced an isolated RLNR as a first failure and received salvage treatment. Patients and tumor characteristics of both primary and recurrent diseases were collected after obtaining the approval of the Institutional Review Board of each institution.

Ethical approval and Informed consent. This study was performed in compliance with the principles of the Declaration of Helsinki (1964) and approved by the Institutional Review Board of Seoul National University Hospital and Seoul Metropolitan Government Seoul National University Boramae Medical Center. The informed consent was waived due to the retrospective nature of the study.

Treatment. Salvage treatments after isolated RLNR were variously performed (Table I). As an optimal treatment policy was not established, there was heterogeneity in the sequence of treatments. All patients except one underwent surgical removal of RLNR. The mean number of excised LNs was 7 [interquartile range $(\mathrm{IQR})=2$ 16]. After removal, either or both adjuvant chemotherapy (CT) and RT was delivered to 30 patients. CT alone or sandwich CT surgical removal was done in one patient, respectively. The most commonly used chemotherapy was interferon- $\alpha$ treatment which was administered in 18 patients at different doses and cycles. Dacarbazine-based CT was used in seven patients, and one patient received a vemurafenib. Twelve patients received adjuvant RT, which was delivered mainly using a 3-dimensional RT technique (10 patients). The median RT dose was 40 Gy (range=30-54 Gy) with daily fractions of 1.8 to $5.0 \mathrm{~Gy}$.
Table I. Salvage treatments for isolate RLNR.

\begin{tabular}{lc}
\hline Sequence of treatment & $\mathrm{N}(\%)$ \\
\hline $\mathrm{OP}$ & $8(20.0)$ \\
$\mathrm{OP} \rightarrow \mathrm{CT}$ & $18(45.0)$ \\
$\mathrm{OP} \rightarrow \mathrm{RT}$ & $6(15.0)$ \\
$\mathrm{OP} \rightarrow \mathrm{CT} \rightarrow \mathrm{RT}$ & $3(7.5)$ \\
$\mathrm{OP} \rightarrow \mathrm{RT} \rightarrow \mathrm{CT}$ & $3(7.5)$ \\
$\mathrm{CT}$ & $1(2.5)$ \\
$\mathrm{CT} \rightarrow \mathrm{OP} \rightarrow \mathrm{CT}$ & $1(2.5)$ \\
\hline
\end{tabular}

RLNR: Regional lymph node recurrence; OP: operation; CT: chemotherapy; RT: radiotherapy.

Immunohistochemistry. Immunohistochemistry was performed to examine the prognostic impact of FOXP3, pAkt, pS6, and VEGF in patients with available tumor tissue samples (28/40 patients). The immunostaining of formalin-fixed paraffin embedded tissues was performed using an automated immunostainer (Ventana BenchMark ULTRA, Ventana Medical System Inc., Tucson, AZ, USA) according to the manufacturer's protocol. The primary antibodies used for staining were as follows: FOXP3 (236A/E7, Abcam, Cambridge, UK), VEGF (MAB293, R\&D systems, Minneapolis, MN, USA), phospho-AKT (Ser473, 4060S, Cell Signaling Technology, Danvers, MA, USA), and pS6 (Ser240/244, 5464S, Cell Signaling Technology, Danvers, MA, USA).

Tissues were homogenously stained for FOXP3 and VEGF and characterized as negative (no stain or weak stain) or positive (moderate or strong staining) depending on the staining intensity. For pAkt, scoring was based on a 3-point scale (11) incorporating both staining intensity and stained cell rates. Positivity was defined as a score of 2 or 3 points, and the nucleus and cytoplasm were scored separately. For pS6, the protein was stained in the cytoplasm with relatively uniform intensity. A negative score was defined as a staining rate $<20 \%$ and positive as that of $\geq 20 \%$.

Variables and statistical analysis. All primary cancers were restaged according to the AJCC 8th cancer staging manual (12). The initial resection margin was categorized as negative, close (not involved, but a margin less than $1 \mathrm{~cm}$ ), and positive (tumor on ink). The LN ratio (LNR) was calculated by dividing the NMLN by the number of harvested LNs (NHLN). To avoid confusion, the NHLN at the first diagnosis of malignant melanoma was denoted by iNHLN and the NHLN at the time of recurrence was denoted by rNHLN. In addition, the NMLN at the first diagnosis was expressed as iNMLN and the NMLN at the time of recurrence was expressed as rNMLN.

The disease-free interval (DFI) was the duration from the diagnosis of primary malignant melanoma to the detection of isolated RLNR. After isolated RLNR, the following clinical endpoints were calculated from the diagnosis of recurrence to the last follow-up, detection of failure, or death from any cause: postrecurrence recurrence-free survival (pRFS) and post-recurrence overall survival (pOS).

Survival estimation was performed using the Kaplan-Meier method and the survival difference between categorical variables was compared by log-rank test. The continuous variables were fitted to the Cox proportional hazards model after verification of the 
Table II. Patient and primary tumor characteristics.

\begin{tabular}{|c|c|}
\hline Characteristics & $\mathrm{N}(\%)$ \\
\hline \multicolumn{2}{|l|}{ Age at primary diagnosis } \\
\hline Median (range), years & $53(18-77)$ \\
\hline \multicolumn{2}{|l|}{ Gender } \\
\hline Male & $24(60.0)$ \\
\hline Female & $16(40.0)$ \\
\hline \multicolumn{2}{|l|}{ Primary site } \\
\hline Head and neck & $1(2.5)$ \\
\hline Upper extremity & $10(25.0)$ \\
\hline Lower extremity & $26(65.0)$ \\
\hline Trunk & $2(5.0)$ \\
\hline Unknown & $1(2.5)$ \\
\hline \multicolumn{2}{|l|}{ Initial $\mathrm{T}$ stage $^{\mathrm{a}}$} \\
\hline $\mathrm{T} 1-2$ & $8(20.0)$ \\
\hline $\mathrm{T} 3-4$ & $19(47.5)$ \\
\hline \multicolumn{2}{|l|}{ Initial invasion level ${ }^{\mathrm{a}}$} \\
\hline Clark level II & $3(7.5)$ \\
\hline Clark level III & $1(2.5)$ \\
\hline Clark level IV & $9(22.5)$ \\
\hline Clark level V & $11(27.5)$ \\
\hline \multicolumn{2}{|l|}{ Initial $\mathrm{N}$ stage $\mathrm{a}^{\mathrm{a}}$} \\
\hline No & $27(67.5)$ \\
\hline N1-2 & $7(17.5)$ \\
\hline N3 & $5(12.5)$ \\
\hline \multicolumn{2}{|l|}{ Initial ulceration ${ }^{\mathrm{a}}$} \\
\hline Absent & $17(42.5)$ \\
\hline Present & $10(25.0)$ \\
\hline \multicolumn{2}{|c|}{ Initial lymphovascular invasiona } \\
\hline Absent & $15(3.5)$ \\
\hline Present & $4(10.0)$ \\
\hline \multicolumn{2}{|l|}{ Initial resection margin ${ }^{a}$} \\
\hline Negative & $10(25.0)$ \\
\hline Close/positive & $14(35.0)$ \\
\hline \multicolumn{2}{|c|}{ Initial surgical LN evaluation } \\
\hline Not done & $28(70.0)$ \\
\hline Excision & $5(12.5)$ \\
\hline Sentinel biopsy & $2(5.0)$ \\
\hline LN dissection & $5(12.5)$ \\
\hline \multicolumn{2}{|c|}{ No. of initially examined LNs } \\
\hline Mean (IQR) & $4(0-5)$ \\
\hline \multicolumn{2}{|c|}{ No. of initially metastatic LNs } \\
\hline Mean (IQR) & $2(0-2)$ \\
\hline
\end{tabular}

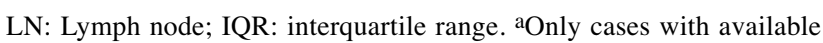
information were analyzed.

proportional hazards assumption. Multivariate analyses were only carried out for pRFS due to the limited number of deaths. A $p$-value $<0.05$ was considered statistically significant. All statistical analyses were performed using R version 3.4.0 (R Foundation for Statistical Computing, Vienna, Austria, http://www.r-project.org).

\section{Results}

Characteristics. Patient and tumor characteristics at the time of primary diagnosis are shown in Table II. The median age of the patients was 53 years and the male to female ratio was
Table III. Characteristics of recurrent disease.

\begin{tabular}{lc}
\hline Characteristics & $\mathrm{N}(\%)$ \\
\hline Age at recurrence & \\
$\quad$ Median (range), years & $55(20-78)$ \\
Basins of LN recurrence & \\
$\quad$ Head and neck & $1(2.5)$ \\
Axilla & $12(30.0)$ \\
Groin/iliac & $27(67.5)$ \\
Extent of surgical removal & \\
Not done & $1(2.5)$ \\
Excision & $17(42.5)$ \\
LN dissection & $22(55.0)$ \\
No. of examined LNs & \\
Mean (IQR) & $10(2-16)$ \\
No. of metastatic LNs & \\
Mean (IQR) & $4(1-6)$ \\
The greatest size of recurred LNs & \\
$\leq 3 \mathrm{~cm}$ & $24(60.0)$ \\
$3-6 \mathrm{~cm}$ & $13(32.5)$ \\
$>6 \mathrm{~cm}$ & $2(5.0)$ \\
Chemotherapy & \\
Done & $14(35.0)$ \\
Not done & $26(65.0)$ \\
Radiotherapy & \\
Done & $28(70.0)$ \\
Not done & $12(30.0)$ \\
Disease-free interval & \\
$\leq 12$ months & $19(47.5)$ \\
$>12$ months & $21(52.5)$ \\
\hline
\end{tabular}

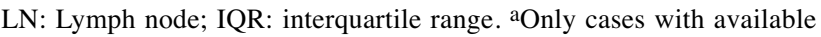
information were analyzed.

3:2. The lower extremity was the most common primary site $(65.0 \%)$. Although the information on the primary tumor stage and depth of invasion were not available for all patients, the proportion of patients with T3-4 stage were greater than those with $\mathrm{T} 1-2 \quad(47.5 \%$ and $20.0 \%$, respectively). The majority of patients $(70.0 \%)$ did not undergo surgical LN evaluation. For the 12 patients who had undergone LN evaluation, the mean iNHLN and iNMLN were $4(\mathrm{IQR}=0-5)$ and $2(\mathrm{IQR}=0-2)$, respectively.

The median DFI was 12 months. At the time of isolated RLNR, the median age of the patients was 55 years. The groin or iliac was the most common site of $\mathrm{LN}$ recurrence in 27 patients. The excision and dissection of the LNs were performed in 17 and 22 patients, respectively. The mean rNMLN was four (IQR=1-6) and the mean size of the greatest recurred $\mathrm{LN}$ was $2.8 \mathrm{~cm}(\mathrm{IQR}=2-3.3)$. Disease characteristics and treatment at the time of recurrence are depicted in Tables I and III.

Patterns of failure after isolated RLNR. During follow-up (median, 28 months; range=3-224 months), only 8 patients had maintained a disease-free status after salvage treatment. 
Table IV. Clinical factors affecting the disease-free interval.

\begin{tabular}{|c|c|c|c|}
\hline Clinical factor & $\begin{array}{c}\text { DFI } \leq 12 \mathrm{mo} \\
\mathrm{N}\end{array}$ & $\begin{array}{c}\text { DFI }>12 \text { mo } \\
\mathrm{N}\end{array}$ & $p$-Value ${ }^{\mathrm{a}}$ \\
\hline \multicolumn{4}{|c|}{ Age at primary diagnosis } \\
\hline$<60$ years & 9 & 20 & \multirow[t]{2}{*}{0.001} \\
\hline$\geq 60$ years & 10 & 1 & \\
\hline \multicolumn{4}{|l|}{ Gender } \\
\hline Male & 11 & 13 & \multirow[t]{2}{*}{$0.796^{\mathrm{b}}$} \\
\hline Female & 8 & 8 & \\
\hline \multicolumn{4}{|l|}{ Primary site* } \\
\hline Head and neck & 1 & 0 & \multirow[t]{5}{*}{0.182} \\
\hline Upper extremity & 3 & 7 & \\
\hline Lower extremity & 14 & 12 & \\
\hline Trunk & 0 & 2 & \\
\hline unknown & 1 & 0 & \\
\hline \multicolumn{4}{|l|}{ T stage* } \\
\hline $\mathrm{T} 1-2$ & 6 & 2 & \multirow[t]{2}{*}{0.236} \\
\hline T3-4 & 9 & 10 & \\
\hline \multicolumn{4}{|l|}{ Clark level* } \\
\hline Clark level II & 1 & 2 & \multirow[t]{4}{*}{0.570} \\
\hline Clark level III & 0 & 1 & \\
\hline Clark level IV & 6 & 3 & \\
\hline Clark level V & 6 & 5 & \\
\hline \multicolumn{4}{|l|}{ Ulceration* } \\
\hline Absent & 10 & 7 & \multirow[t]{2}{*}{0.706} \\
\hline Present & 5 & 5 & \\
\hline \multicolumn{4}{|c|}{ Lymphovascular invasion* } \\
\hline Absent & 9 & 6 & \multirow[t]{2}{*}{0.255} \\
\hline Present & 4 & 0 & \\
\hline \multicolumn{4}{|l|}{$\mathrm{N}$ stage } \\
\hline No & 9 & 18 & \multirow[t]{2}{*}{0.006} \\
\hline $\mathrm{N} 1-3$ & 10 & 2 & \\
\hline \multicolumn{4}{|c|}{ No of initial harvested LNs } \\
\hline Mean (SD) & $8.2( \pm 13.5)$ & $0.6( \pm 1.7)$ & $0.027^{\mathrm{c}}$ \\
\hline$<7$ & 11 & 17 & \multirow[t]{2}{*}{0.018} \\
\hline$\geq 7$ & 6 & 0 & \\
\hline \multicolumn{4}{|c|}{ No of initial metastatic LNs } \\
\hline Mean (SD) & $3.4( \pm 8)$ & $1.9( \pm 6)$ & $0.111^{\mathrm{c}}$ \\
\hline$<4$ & 14 & 17 & \multirow[t]{2}{*}{0.047} \\
\hline$\geq 4$ & 5 & 0 & \\
\hline
\end{tabular}

DFI: Disease-free interval; LN: lymph node; SD: standard deviation; mo: months. *Only cases with available information were analyzed. ${ }^{\mathrm{a}} p$ value by Fisher's exact test; ${ }^{b} p$-value by $\chi^{2}$ test; ${ }^{c} p$-value by $t$-test.

The most common failure pattern after isolated RLNR was distant metastasis, which occurred in 27 patients. More than half of the distant metastases were accompanied by regional metastasis (14/27 cases). The most common site of distant failure was lung (12/27 cases) followed by distant LN (7/27 cases). Recurrence in bone, liver, and other intraabdominal visceral organs was observed in 5 patients. Secondary regional failures were detected in $50 \%$ of the patients. Local failure was uncommon $(12.5 \%, 5$ patients) and all cases were accompanied by regional failure with or without distant failure (Figure 1).

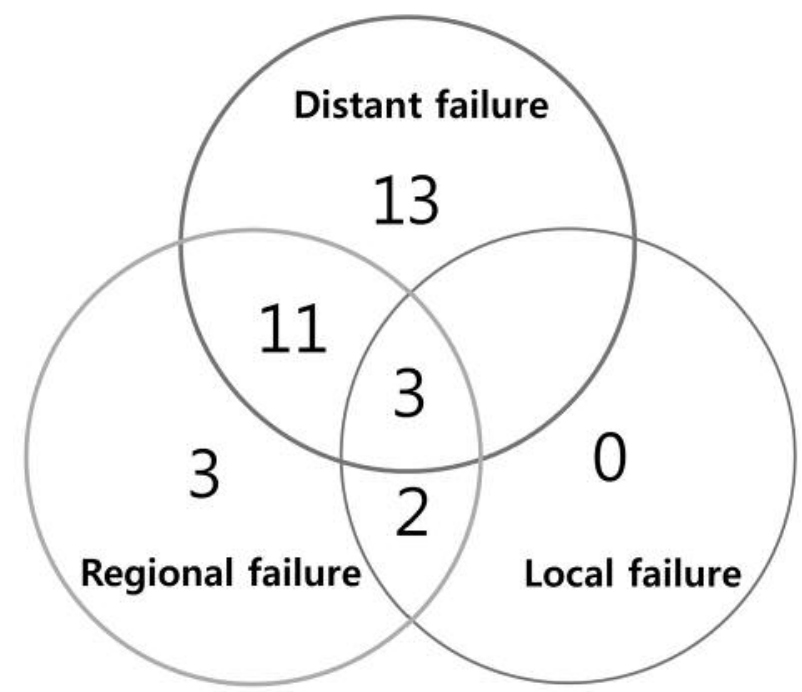

Figure 1. Patterns of failure after isolated regional lymph node recurrence.

Survival analysis and prognosticators. First, we analyzed which group of patients experienced an earlier isolated RLNR. Patients were divided into two groups based on the median DFI value: short-DFI ( $\leq 12$ months) or long-DFI $(>12$ months). There was a significantly higher proportion of patients who were 60 years or older $(p=0.001)$ and those with positive metastatic LNs $(p=0.006)$ in the short-DFI group. The iNHLN was significantly higher in the short-DFI group (mean, $8 v s .1, p=0.027$ ). The comparison of the shortDFI and long-DFI groups is shown in Table IV.

Next, we conducted a post-recurrence survival analysis (Table V). In contrast to DFI, neither age at diagnosis nor age at recurrence contributed to prognosis. The pRFS was significantly decreased according to the iNHLN $(p=0.001)$, and iNMLN $(p=0.007)$. The binomial variable of these two factors also significantly affected pRFS (iNHLN $<7 v s . \geq 7$, $p<0.0001$; iNMLN, $<4 v s . \geq 4, p=0.021)$. However, neither rNHLN $(p=0.074)$ nor rNMLN $(p=0.673)$ was associated with pRFS, a finding that was consistent even when the analysis was performed by replacing each of them with a categorical variable. In contrast, the LNR at the time of recurrence significantly influenced pRFS ( $p=0.008$, Figure 2$)$. A short DFI ( $\leq 12$ months) was also significantly associated with decreased pRFS ( $p=0.009$, Figure $3 \mathrm{~A})$. There was no difference in the pRFS according to the salvage treatment modalities. Multivariate analysis was performed for pRFS. After adjusting the interactions between covariates, an iNHLN $>7 \quad(p=0.005)$ and LNR $>22.2 \%$ at recurrence $(p=0.05)$ were significant prognosticators for pRFS.

In contrast to the poor 1-year pRFS (40\%), the 1-year and 5 -year pOS was $100 \%$ and $85 \%$, respectively. The median 


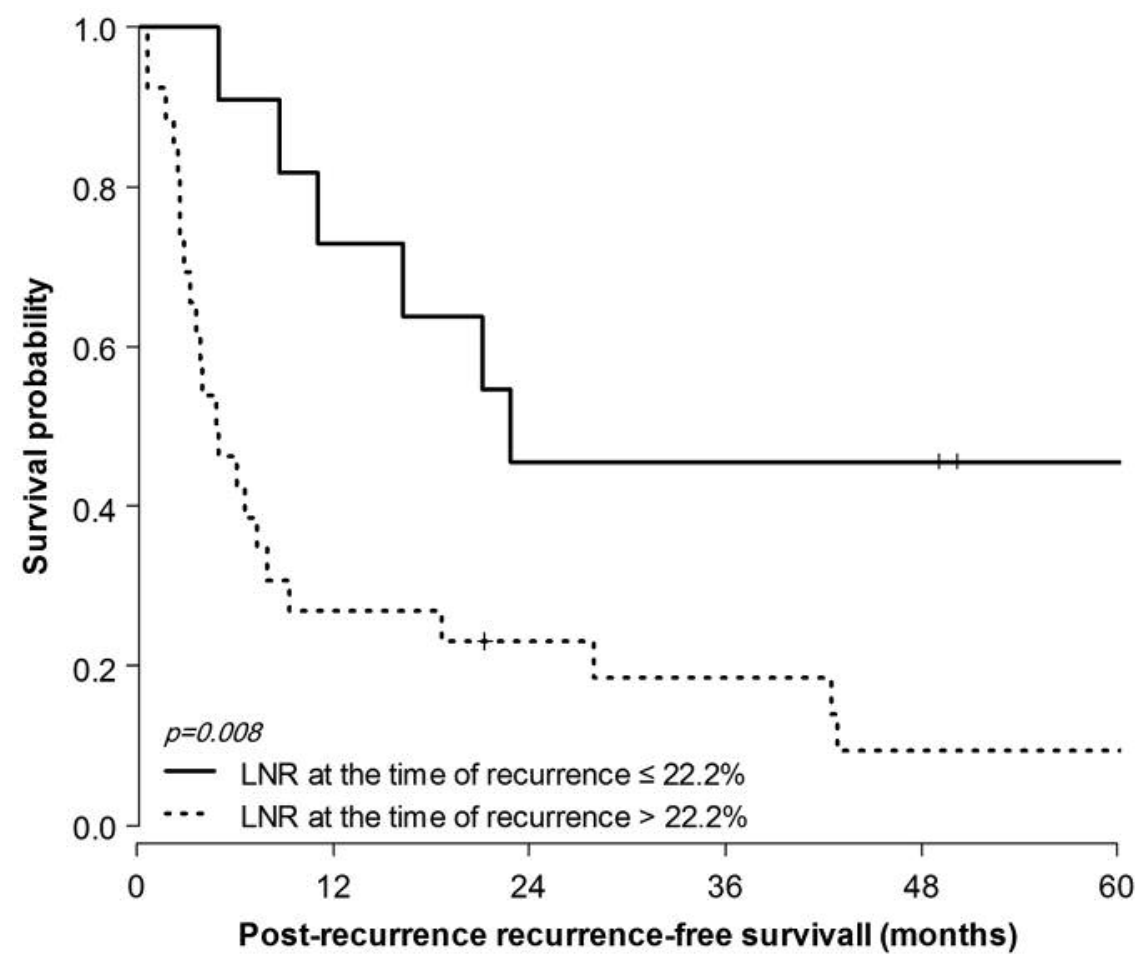

Figure 2. Post-recurrence recurrence-free survival according to lymph node ratio (LNR) at the time of recurrence.

survival time was not reached. Because of the limited number of deaths $(n=5)$, it was difficult to show the survival difference according to a specific variable, except for the DFI. Patients in the short-DFI group (DFI $\leq 12$ months) showed lower pOS than those in the long-DFI group (DFI $>12$ months) ( $p=0.033$, Figure 3B).

After analyzing FOXP3, VEGF, pAkt, and pS6 expression in isolated RLNR, it was revealed that none of these proteins were significant in our study (Table VI).

\section{Discussion}

In this study, we explored the survival outcomes of salvage treatment after isolated RLNR of malignant melanoma. The median pRFS was only 7.2 months and overall survival was 8.6 months even after LN dissection. Once isolated RLNR occurred, subsequent distant failure or locoregional failure was observed in more than $40 \%$ of patients within 1 year. Despite the relatively short pRFS, the 5-year OS rate was $84.9 \%$.

Many studies have reported the prognostic factors that could predict LN recurrence of malignant melanoma. However, there are few studies that have reported the outcome after RLNR. Chao et al. reported follow-up data of 1183 patients after sentinel LN biopsy (13). Recurrence had occurred in 94 patients $(7.9 \%)$ at a median follow-up of 16 months and isolated RLNR only in 12 patients $(1.0 \%)$. This study also reported the factors for predicting recurrence, but there was no analysis of the disease course after recurrence. Eighty-three recurrences had occurred during a median follow-up of 61 months in a study by Jones et al., which analyzed 515 patients with negative sentinel LN (14). Regional recurrence had occurred in 21 patients $(4.0 \%)$ and 11 of them died within the follow-up period. The 5-year OS rate after any recurrence was $68 \%$. The study showed that the survival rate of patients with distant recurrence differed significantly according to the location of the first detected distant recurrence, but the survival analysis for the regional recurrence group was not reported separately.

It is well known that the risk of RLNR increases with the iNMLN $(6,7,13)$. Although all the patients included in our study experienced RLNR, the primary DFI was significantly different according to the iNMLN (data not shown; median DFI of 16 months in patients without metastatic LN, 8 months in patients with 1 3 metastatic LNs, and 6 months in patients with $>3$ metastatic LNs $[p=0.0004])$. The iNMLN was also significantly associated with pRFS in the univariate analysis. However, the rNMLN was not associated with pRFS.

Not only iNMLN but also iNHLN was reported as a predictor of both RLNR and OS (7, 15). Agrawal et al. demonstrated that the iNHLN was significantly associated 
Table V. Univariate survival analysis.

\begin{tabular}{|c|c|c|c|c|c|}
\hline Characteristics & $\mathrm{N}(\%)$ & 1 -year pDFS $(\%)$ & $p$-Value $^{a}$ & 1 -year pOS (\%) & $p$-Value \\
\hline 1-year overall probability & & 40.0 & & 100.0 & \\
\hline \multicolumn{6}{|l|}{ Age at primary diagnosis } \\
\hline$<60$ years & $29(72.5)$ & 41.4 & 0.688 & 96.3 & 0.890 \\
\hline$\geq 60$ years & $11(27.5)$ & 36.4 & & 100.0 & \\
\hline \multicolumn{6}{|l|}{ Gender } \\
\hline Male & $24(60.0)$ & 41.7 & 0.993 & 95.2 & 0.242 \\
\hline Female & $16(40.0)$ & 37.5 & & 100.0 & \\
\hline \multicolumn{6}{|l|}{ Primary T stage* } \\
\hline $\mathrm{T} 1-2$ & 8 (29.6) & 37.5 & 0.687 & 100.0 & 0.365 \\
\hline T3-4 & $19(70.4)$ & 42.1 & & 94.4 & \\
\hline \multicolumn{6}{|l|}{ Primary $\mathrm{N}$ stage } \\
\hline No & $27(69.2)$ & 44.4 & 0.198 & 100.0 & 0.827 \\
\hline N1-3 & $12(30.8)$ & 25.0 & & 90.0 & \\
\hline \multicolumn{6}{|l|}{ Primary margin status* } \\
\hline Negative & $10(41.7)$ & 40.0 & 0.541 & 100.0 & 0.893 \\
\hline close/positive & $14(58.3)$ & 42.9 & & 100.0 & \\
\hline \multicolumn{6}{|l|}{ No of initial harvested LNs } \\
\hline HR $(95 \% \mathrm{CI})$ & & $1.08(1.03-1.13)$ & $0.001^{\mathrm{b}}$ & $1.07(0.94-1.22)$ & $0.317^{\mathrm{b}}$ \\
\hline$<7$ & $17(82.3)$ & 50.0 & $<0.001$ & 100.0 & 0.180 \\
\hline$\geq 7$ & $6(17.7)$ & 0 & & 75.0 & \\
\hline \multicolumn{6}{|l|}{ No of initial metastatic LNs } \\
\hline HR $(95 \% \mathrm{CI})$ & & $1.11(1.03-1.20)$ & $0.007^{b}$ & $0.63(0.17-2.27)$ & $0.479^{\mathrm{b}}$ \\
\hline$<4$ & $31(86.1)$ & 41.9 & 0.021 & 96.6 & 0.659 \\
\hline$\geq 4$ & $5(13.9)$ & 20.0 & & 100.0 & \\
\hline \multicolumn{6}{|l|}{ Age at recurrence diagnosis } \\
\hline$<60$ years & $27(67.5)$ & 40.7 & 0.964 & 96.0 & 0.747 \\
\hline$\geq 60$ years & $13(32.5)$ & 38.5 & & 100.0 & \\
\hline \multicolumn{6}{|l|}{ Disease free interval } \\
\hline$\leq 12$ months & $19(47.5)$ & 21.1 & 0.009 & 93.3 & 0.033 \\
\hline$>12$ months & $21(52.5)$ & 57.1 & & 100.0 & \\
\hline \multicolumn{6}{|l|}{ Type of surgical removal } \\
\hline Excision & $17(42.5)$ & 29.4 & 0.399 & 100.0 & 0.864 \\
\hline LN dissection & $22(55.0)$ & 50.0 & & 95.2 & \\
\hline \multicolumn{6}{|l|}{ No of harvested LNs } \\
\hline HR $(95 \% \mathrm{CI})$ & & $0.96(0.92-1.00)$ & $0.074^{\mathrm{b}}$ & $0.99(0.91-1.08)$ & $0.809^{\mathrm{b}}$ \\
\hline$\leq 7$ & $21(55.3)$ & 33.3 & 0.221 & 94.1 & 0.688 \\
\hline$>7$ & $17(44.7)$ & 47.1 & & 100.0 & \\
\hline \multicolumn{6}{|l|}{ No of metastatic LNs } \\
\hline HR $(95 \% \mathrm{CI})$ & & $1.01(0.95-1.08)$ & $0.673^{b}$ & $1.07(0.96-1.19)$ & $0.217^{\mathrm{b}}$ \\
\hline$\leq 2$ & $24(61.5)$ & 45.8 & 0.509 & 95.2 & 0.615 \\
\hline$>2$ & $15(38.5)$ & 33.3 & & 100.0 & \\
\hline \multicolumn{6}{|l|}{$\mathrm{LN}$ ration at recurrence } \\
\hline$\leq 22.2 \%$ & $11(29.7)$ & 72.7 & 0.008 & 90.9 & 0.434 \\
\hline$>22.2 \%$ & $26(70.3)$ & 26.9 & & 95.5 & \\
\hline \multicolumn{6}{|l|}{ Size of recurred LN } \\
\hline$\leq 3 \mathrm{~cm}$ & $24(61.5)$ & 37.5 & 0.741 & 57.1 & 0.261 \\
\hline $3-6 \mathrm{~cm}$ & $13(33.3)$ & 53.9 & & 84.6 & \\
\hline$\geq 6 \mathrm{~cm}$ & $2(5.1)$ & 0.0 & & 0.0 & \\
\hline \multicolumn{6}{|l|}{ Salvage chemotherapy } \\
\hline Not done & $14(35.0)$ & 35.7 & 0.760 & 91.7 & 0.653 \\
\hline Done & $26(65.0)$ & 42.3 & & 100.0 & \\
\hline \multicolumn{6}{|l|}{ Salvage radiotherapy } \\
\hline Not done & $30(75.0)$ & 42.9 & 0.431 & 100.0 & 0.070 \\
\hline Done & $10(25.0)$ & 33.3 & & 90.9 & \\
\hline
\end{tabular}

pDFS: Post-recurrence disease-free survival; pOS: post-recurrence overall survival; HR: hazard ratio; CI: confidence interval; LN: lymph node.

*Only cases with available information were analyzed. ${ }^{a} p$-value by Log-rank test; ${ }^{\mathrm{b}} p$-value by Cox proportional hazards model. 

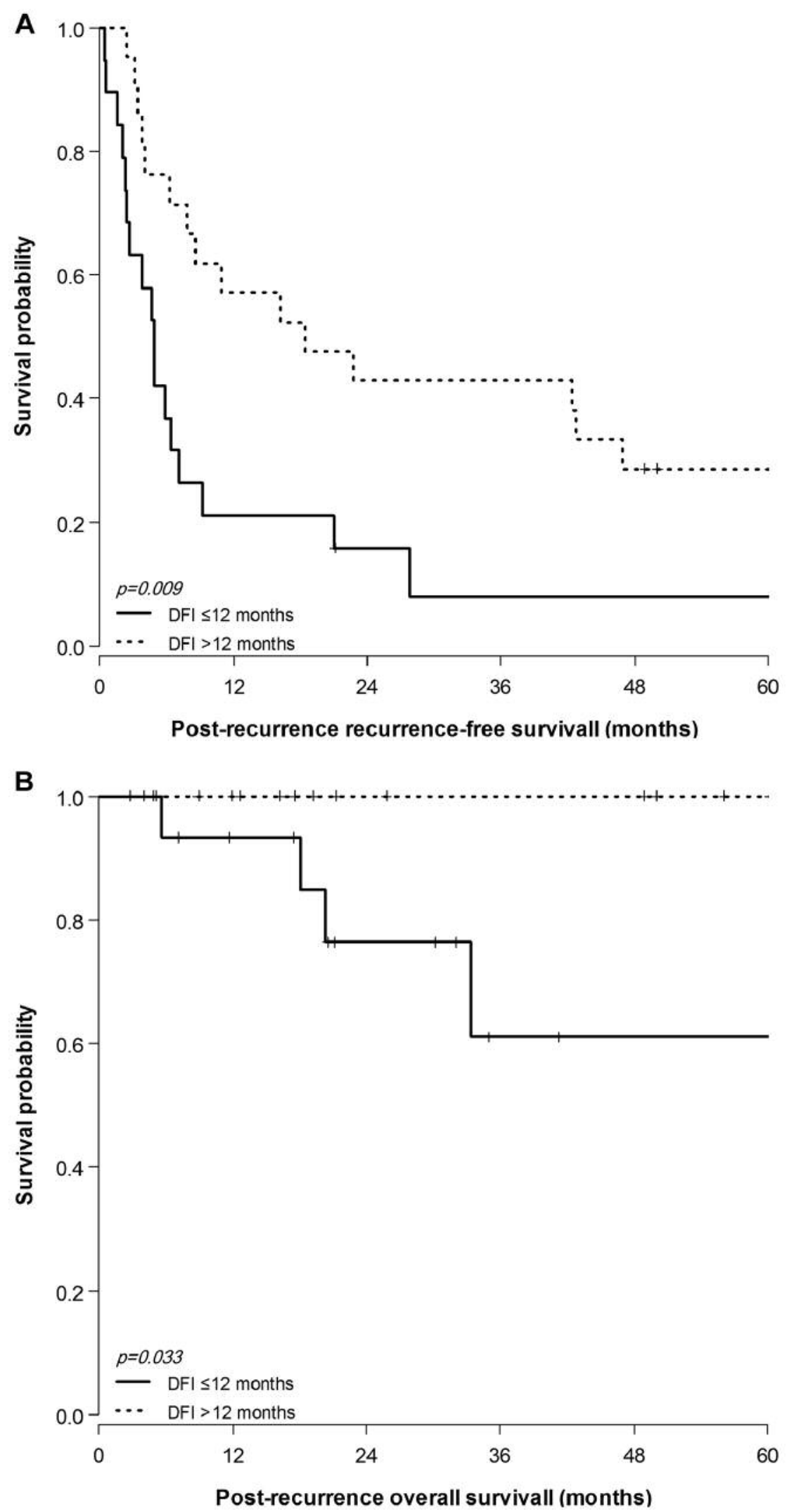

Figure 3. Post-recurrence recurrence-free survival (A) and post-recurrence overall survival (B) according to primary disease-free interval (DFI). 
Table VI. Univariate analysis of immunohistochemistry.

\begin{tabular}{|c|c|c|c|c|c|}
\hline Characteristics & $\mathrm{N}(\%)$ & 1 -year pDFS (\%) & $p$-Value $^{\mathrm{a}}$ & 1 -year pOS (\%) & $p$-Value \\
\hline \multicolumn{6}{|l|}{ FOXP3 } \\
\hline Negative & 15 (53.6) & 40.0 & \multirow[t]{2}{*}{0.307} & 100.0 & \multirow[t]{2}{*}{0.660} \\
\hline Positive & $13(46.4)$ & 46.2 & & 100.0 & \\
\hline \multicolumn{6}{|l|}{ VEGF } \\
\hline Negative & $3(10.7)$ & 33.3 & \multirow[t]{2}{*}{0.384} & 100.0 & \multirow[t]{2}{*}{0.337} \\
\hline Positive & $25(89.3)$ & 44.0 & & 100.0 & \\
\hline \multicolumn{6}{|l|}{ pAkt, cytoplasmic } \\
\hline Negative & $5(17.9)$ & 20.0 & \multirow[t]{2}{*}{0.179} & 100.0 & \multirow[t]{2}{*}{0.263} \\
\hline Positive & $23(82.1)$ & 47.8 & & 100.0 & \\
\hline \multicolumn{6}{|l|}{ pAkt, nuclear } \\
\hline Negative & $20(71.4)$ & 50.0 & \multirow[t]{2}{*}{0.570} & 100.0 & \multirow[t]{2}{*}{0.621} \\
\hline Positive & 8 (28.6) & 25.0 & & 100.0 & \\
\hline \multicolumn{6}{|l|}{$\mathrm{pS} 6$} \\
\hline Negative $(\leq 20 \%)$ & $23(82.1)$ & 43.5 & \multirow[t]{2}{*}{0.696} & 100.0 & \multirow[t]{2}{*}{0.263} \\
\hline Positive $(>20 \%)$ & $5(17.9)$ & 40.0 & & 100.0 & \\
\hline
\end{tabular}

pDFS: Post-recurrence disease-free survival; pOS: post-recurrence overall survival; FOXP3: forkhead box P; VEGF: vascular endothelial growth factor. ${ }^{\mathrm{a}} p$-value by Log-rank test.

with RLNR and disease-specific survival in 615 patients who had underwent therapeutic lymphadenectomy for clinically advanced, LN metastatic melanoma (7). In our study, the iNHLN was significantly associated with the pRFS. However, the result does not seem to indicate the absolute importance of the iNHLN. The number of patients with an iNHLN $\geq 7$ was relatively small (6 patients, $17.7 \%$ ). All patients had an iNMLN $\geq 3$ and a DFI $<12$ months. Patients who already had poor prognostic factors at the time of initial diagnosis had an iNHLN $\geq 7$. Similar to the rNMLN, pRFS was not significantly increased by removing more LNs after RLNR.

LNR is considered an important prognosticator in various solid tumors (16-18). The study by Xing et al. using the Surveillance, Epidemiology, and End Results database showed that the LNR was significantly associated with disease-specific survival in melanoma patients (19). LNR has the advantage of simultaneously considering the NHLN and the NMLN. When the surgical evaluation of LNs is limited, simply assessing the NMLNs poses a risk of underestimating the regional tumor burden. In contrast, the LNR can reflect the likelihood of finding a metastatic LN depending on the extent of removal. In the present study, the LNR at the time of RLNR was an important prognostic factor in multivariate analysis, although neither the rNHLN nor rNMLN had a statistically significant association with survival. For example, if two $\mathrm{LN}$ metastases were found, the patients are always rpN1 or NMLN 2. However, when evaluated by LNR, patients with an NHLN $>8$ are classified as low-risk (LNR $\leq 22.2 \%$ ) and patients with an NHLN $\leq 8$ (LNR $>22.2 \%$ ) are classified as high-risk. Because our study included a relatively small number of patients, we cannot conclude from these results that the rNHLN or rNMLN does not affect prognosis after RLNR. However, despite the small number of patients, the LNR clearly showed a clinical relevance in multivariate analysis. In particular, there are limited opportunities for the complete re-dissection of the $\mathrm{LN}$ area at the time of recurrence due to technical difficulties or treatment-related toxicity. Therefore, the LNR at the time of recurrence could be more clinically valuable.

We tried to explore whether aggressive surgery or adjuvant RT would be helpful in preventing the progression of disease after RLNR. However, all patients except one had surgical resection of RLNR, and adjuvant RT was only performed in 12 patients. In terms of surgical extent, LN dissection did not affect the prognosis, but LNR at salvage surgery could be an important prognostic index, as mentioned above. Although the number of patients was very small, the addition of adjuvant RT did not change the clinical outcomes in this study. Even if isolated RLNR occurred as a first recurrence, one third of patients eventually experienced distant failure within 9 months and the median post-recurrence distant failure-free survival was 18 months, compared with 22 months for the median post-recurrence locoregional failure-free survival. Therefore, we assume that even the isolated RLNR should be approached as systemic disease rather than locoregional disease.

We performed immunohistochemical staining to find a biomarker to help predict the prognosis of the patient, but unfortunately did not yield meaningful results. Immunosuppression in the tumor microenvironment mediated by regulatory $\mathrm{T}$ cell (Treg) acts as a major mechanism in tumor immune escape, which is considered to 
be the major hurdle of immunotherapy. The accumulation of Treg was reported to be associated with the survival of malignant melanoma patients (20). In a study with 185 primary malignant melanoma patients, Gerber et al. reported that the DFI and OS were significantly increased in patients with low expression of the Treg expression marker FOXP3 (21). In contrast to this, Ladányi et al. found that there was no correlation between the infiltration of FOXP3 and any clinicopathologic parameters, metastasis formation, and survival rate (22). Other investigators also reported conflicting results on the prognostic value of FOXP3 and, even if FOXP3 was associated with prognosis, it was reported as contributing more to either distant failure or to local recurrence (21-24). In our study, there was no difference in prognosis according to the expression levels of FOXP3 in the metastatic LNs. The effect of FOXP3 on the biological behavior of primary or recurrent melanoma requires further investigation.

In the anti-tumor immunity, VEGF has been reported to be a promoter of Treg activation. A prospective study demonstrated a positive correlation between serum VEGF and Treg levels in patients with stage III/IV melanoma, although only a small number of patients were included (25). The study by Rajabi et al. showed a significant association between VEGF expression and Breslow's depth $(p<0.001)$ or Clark's level $(p=0.002)$. However, the analysis of the clinical outcomes was not available in this study (26). A few studies have reported that increased serum VEGF levels are associated with poor outcome in patients with malignant melanoma $(27,28)$. In the present study, there was no difference in survival according to VEGF expression in the metastatic LNs. All but three patients showed moderate to strong staining, which made the comparison between the weak versus strong staining groups difficult. The biased distribution of VEGF expression may reflect the selection bias because all the patients included in this study already had LN recurrence. Further research is needed as the prognostic role of VEGF has not been actively studied in malignant melanoma patients.

The high prevalence of BRAF and NRAS mutations and the clinical activity of the BRAF inhibitor suggest that RASRAF-MEK-ERK pathway plays an important role in malignant melanoma. Mutant NRAS can activate the PI3KAkt pathway as well as the RAS-RAF-MEK-ERK pathway. Dai et al. conducted a study with 170 cases of primary melanoma and showed that the strong expression of phosphorylated AKT was significantly more frequent in invasive melanoma than in normal nevi, and was more frequent in stage II-III than stage I. They also reported a significant inverse correlation between pAKT expression and OS $(p=0.049)$ or DFI $(p=0.014)$. In their subgroup analysis according to tumor thickness, there was a significant association between strong pAKT expression and poor OS in the low-risk group $(\leq 1.5 \mathrm{~mm})$ but not in the high-risk group ( $>1.5 \mathrm{~mm})(29)$. There was no survival difference according to pAKT expression in our study. This result may be due to the small number of patients; however, it may also be related to the Dai et al. result regarding the lack of association of pAKT expression with poor OS in a high-risk group. It may suggest that pAKT expression is prognostic in the early stages of melanoma but not after progression or relapse. Similarly, in a study analyzing the association of pAKT expression levels and clinical outcomes of patients with regional metastasis, there was no difference in survival according to pAKT levels (30).

The phosphorylation of S6, a component of the mTOR pathway which is downstream of the PI3K/AKT pathway, is an indicator of the AKT/mTOR pathway. Pópulo et al. showed that pS6 is expressed at higher levels in patients with Clark level III/IV compared to those with Clark level I/II. In addition, the increased expression of pS6 was related to poor prognostic features (31). However, survival analysis according to pS6 expression levels had not been conducted in that study. In our study, the pS6 expression was mostly lower in recurred LNs. According to a recent report, pS6 level is a predictive marker of ERK-independent resistance, especially to RAF and MEK inhibitor. The decrease in pS6 expression after treatment predicted significantly increased progression-free survival (32).

The present study is subject to certain limitations stemming from its retrospective nature. There are many cases where information on several important prognostic factors such as Clark level or ulceration is missing. The diversity of patient characteristics and treatment patterns also resulted in heterogeneity in this study. Due to the rarity of the disease, the number of patients included in this study was small. Nevertheless, to our best knowledge, ours is the first study of the prognostic factors after isolated RLNR. In particular, we report for the first time the prognostic significance of LNR at the time of RLNR. In addition, several of the prognostic biomarkers that we analyzed have rarely been studied in these RLNR settings, and the collective findings are of value as a negative result.

\section{Conclusion}

The isolated RLNR occurs mostly within 1-2 years after the first diagnosis of malignant melanoma. Even if isolated regional recurrence occurred, patients will experience a second failure within several months. In our study, the number of initially harvested LNs $>7$ and LNR $>22 \%$ at the time of relapse were found to be poor prognostic factors for pRFS. The development of optimal treatment strategies for these patients is required. Considering the frequent distant failure after regional recurrence, systemic control seems to be 
important for isolated RLNR. Despite the short pRFS, the recent advances in treatment techniques appear to have improved the pOS rate compared to those reported in previous studies. Since most patients survived more than 5 years after recurrence, treatment-related chronic complications should be considered when determining treatment policy.

\section{Conflicts of Interest}

There are no conflicts of interest to declare regarding this study.

\section{Authors' Contributions}

JK analyzed data and wrote an article; YAK designed the work and oversaw the process of making the IHC staining; CL and BHK collected and interpreted data; SK and IHK designed study and edited and finalized the manuscript.

\section{Acknowledgements}

This research was supported by the National Research Foundation of Korea sponsored by the Ministry of Science, ICT \& Future Planning (NRF-2016R1A2B4012095), Republic of Korea.

\section{References}

1 Jemal A, Saraiya M, Patel P, Cherala SS, Barnholtz-Sloan J, Kim J, Wiggins CL and Wingo PA: Recent trends in cutaneous melanoma incidence and death rates in the United States, 19922006. J Am Acad Dermatol 65: S17.e1-S17.e11, 2011. PMID: 22018063. DOI: 10.1016/j.jaad.2011.04.032

2 Morton DL, Thompson JF, Cochran AJ, Mozzillo N, Elashoff R, Essner R, Nieweg OE, Roses DF, Hoekstra HJ, Karakousis CP, Reintgen DS, Coventry BJ, Glass EC and Wang HJ: Sentinelnode biopsy or nodal observation in melanoma. N Engl J Med 355: 1307-1317, 2006. PMID: 17005948. DOI: 10.1056/ NEJMoa060992

3 National Comprehensive Cancer Network: Cutaneous Melanoma (Version 1.2019), 2018. Available from: https://www.nccn.org/ professionals/physician_gls/pdf/cutaneous_melanoma.pdf

4 Miller EJ, Daly JM, Synnestvedt M, Schultz D, Elder D and Guerry D: Loco-regional nodal relapse in melanoma. Surg Oncol 1: 333-340, 1992. PMID: 1341268. DOI: 10.1016/09607404(92)90033-H

5 Nathansohn N, Schachter J and Gutman H: Patterns of recurrence in patients with melanoma after radical lymph node dissection. Arch Surg 140: 1172-1177, 2005. PMID: 16365238. DOI: 10.1001/archsurg.140.12.1172

6 Pidhorecky I, Lee RJ, Proulx G, Kollmorgen DR, Jia C, Driscoll DL, Kraybill WG and Gibbs JF: Risk factors for nodal recurrence after lymphadenectomy for melanoma. Ann Surg Oncol 8: 109-115, 2001. PMID: 11258774. DOI: 10.1007/ s10434-001-0109-2

7 Agrawal S, Kane JM, Guadagnolo BA, Kraybill WG and Ballo MT: The benefits of adjuvant radiation therapy after therapeutic lymphadenectomy for clinically advanced, high-risk, lymph node-metastatic melanoma. Cancer 115: 5836-5844, 2009. PMID: 19701906. DOI: 10.1002/cncr.24627
8 Henderson MA, Burmeister BH, Ainslie J, Fisher R, Di Iulio J, Smithers BM, Hong A, Shannon K, Scolyer RA, Carruthers S, Coventry BJ, Babington S, Duprat J, Hoekstra HJ and Thompson JF: Adjuvant lymph-node field radiotherapy versus observation only in patients with melanoma at high risk of further lymphnode field relapse after lymphadenectomy (ANZMTG 01.02/TROG 02.01): 6-year follow-up of a phase 3, randomised controlled trial. Lancet Oncol 16: 1049-1060, 2015. PMID: 26206146. DOI: 10.1016/S1470-2045(15)00187-4

9 Rischke HC, Schultze-Seemann W, Wieser G, Krönig M, Drendel V, Stegmaier P, Krauss T, Henne K, Volegova-Neher N, Schlager D, Kirste S, Grosu A-L and Jilg CA: Adjuvant radiotherapy after salvage lymph node dissection because of nodal relapse of prostate cancer versus salvage lymph node dissection only. Strahlenther Onkol 191: 310-320, 2015. PMID: 25326142. DOI: 10.1007/s00066-014-0763-5

10 Yamashita H, Jingu K, Niibe Y, Katsui K, Matsumoto T, Nishina $\mathrm{T}$ and Terahara A: Definitive salvage radiation therapy and chemoradiation therapy for lymph node oligo-recurrence of esophageal cancer: a Japanese multi-institutional study of 237 patients. Radiat Oncol 12: 38, 2017. PMID: 28219406. DOI: 10.1186/s13014-017-0780-5

11An J, Jeong H, Lee Y, Woo SU, Seo JH and Kim A: Phosphorylated Akt and phosphorylated mTOR expression in breast invasive carcinomas: Analysis of 530 cases. J Breast Cancer 13: 337-348, 2010. DOI: 10.4048/jbc.2010.13.4.337

12 Amin MB, Edge SB, Greene FL, Byrd DR, Brookland RK, Washington MK, Gershenwald JE, Compton CC, Hess KR, Sullivan DC, Jessup JM, Brierley JD, Gaspar LE, Schilsky RL, Balch CM, Winchester DP, Asare EA, Madera M, Gress DM and Meyer LR: AJCC Cancer Staging Manual, Springer International Publishing, 2018.

13 Chao C, Wong SL, Ross MI, Reintgen DS, Noyes RD, Cerrito PB, Edwards MJ and McMasters KM: Patterns of early recurrence after sentinel lymph node biopsy for melanoma. Am J Surg 184: 520-524, 2002. PMID: 12488154. DOI: 10.1016/ S0002-9610(02)01102-9

14 Jones EL, Jones TS, Pearlman NW, Gao D, Stovall R, Gajdos C, Kounalakis N, Gonzalez R, Lewis KD, Robinson WA, and McCarter MD: Long-term follow-up and survival of patients following a recurrence of melanoma after a negative sentinel lymph node biopsy result. JAMA Surg 148: 456-461, 2013. PMID: 28219406. DOI: 10.1186/s13014-017-0780-5

15 Chan AD, Essner R, Wanek LA and Morton DL: Judging the therapeutic value of lymph node dissections for melanoma. J Am Coll Surg 191: 16-22; discussion 22-23, 2000. PMID: 10898179. DOI: $10.1016 / \mathrm{S} 1072-7515(00) 00313-6$

16 Tol JAMG, Brosens LAA, van Dieren S, van Gulik TM, Busch ORC, Besselink MGH and Gouma DJ: Impact of lymph node ratio on survival in patients with pancreatic and periampullary cancer. Br J Surg 102: 237-245, 2015. PMID: 25529117. DOI: 10.1002/bjs.9709

17 Yamashita K, Hosoda K, Ema A and Watanabe M: Lymph node ratio as a novel and simple prognostic factor in advanced gastric cancer. Eur J Surg Oncol 42: 1253-1260, 2016. PMID: 27017273. DOI: 10.1016/j.ejso.2016.03.001

18 Prabhu RS, Hanasoge S, Magliocca KR, Hall WA, Chen SA, Higgins KA, Saba NF, El-Deiry M, Grist W, Wadsworth JT, Chen AY and Beitler JJ: Lymph node ratio influence on risk of head and neck cancer locoregional recurrence after initial 
surgical resection: Implications for adjuvant therapy. Head Neck 37: 777-782, 2015. PMID: 24596123. DOI: 10.1002/hed.23662

19 Xing Y, Badgwell BD, Ross MI, Gershenwald JE, Lee JE, Mansfield PF, Lucci A and Cormier JN: Lymph node ratio predicts disease-specific survival in melanoma patients. Cancer 115: 2505-2513, 2009. PMID: 19309746. DOI: 10.1002/ cncr. 24290

20 Jacobs JFM, Nierkens S, Figdor CG, de Vries IJM and Adema GJ: Regulatory $\mathrm{T}$ cells in melanoma: the final hurdle towards effective immunotherapy? Lancet Oncol 13: e32-42, 2012. PMID: 22225723. DOI: 10.1016/S1470-2045(11)70155-3

21 Gerber AL, Münst A, Schlapbach C, Shafighi M, Kiermeir D, Hüsler R and Hunger RE: High expression of FOXP3 in primary melanoma is associated with tumour progression. $\mathrm{Br} \mathrm{J}$ Dermatol 170: 103-109, 2014. PMID: 24116716. DOI: 10.1111/bjd.12641

22 Ladányi A, Mohos A, Somlai B, Liszkay G, Gilde K, Fejos Z, Gaudi I and Tímár J: FOXP3+ cell density in primary tumor has no prognostic impact in patients with cutaneous malignant melanoma. Pathol Oncol Res 16: 303-309, 2010. PMID: 20306313. DOI: 10.1007/s12253-010-9254-X

23 Hillen F, Baeten CIM, van de Winkel A, Creytens D, van der Schaft DWJ, Winnepenninckx V and Griffioen AW: Leukocyte infiltration and tumor cell plasticity are parameters of aggressiveness in primary cutaneous melanoma. Cancer Immunol Immunother 57: 97-106, 2008. PMID: 17602225. DOI: 10.1007/s00262-007-0353-9

24 Miracco C, Mourmouras V, Biagioli M, Rubegni P, Mannucci S, Monciatti I, Cosci E, Tosi P and Luzi P: Utility of tumourinfiltrating $\mathrm{CD} 25+\mathrm{FOXP} 3+$ regulatory $\mathrm{T}$ cell evaluation in predicting local recurrence in vertical growth phase cutaneous melanoma. Oncol Rep 18: 1115-1122, 2007. PMID: 17914561. DOI: $10.3892 /$ or.18.5.1115

25 Agostino NM, Saraceni C, Kincaid H, Shi W, Nevala WK, Markovic S and Nair SG: A prospective evaluation of the role of Vascular Endothelial Growth Factor (VEGF) and the immune system in stage III/IV melanoma. SpringerPlus 4: 186, 2015. PMID: 25932372. DOI: 10.1186/s40064-015-0951-5

26 Rajabi P, Neshat A, Mokhtari M, Rajabi MA, Eftekhari M and Tavakoli P: The role of VEGF in melanoma progression. J Res Med Sci 17: 534-539, 2012. PMID: 23626629.
27 Ascierto PA, Leonardi E, Ottaiano A, Napolitano M, Scala S and Castello G: Prognostic value of serum VEGF in melanoma patients: a pilot study. Anticancer Res 24: 4255-4258, 2004. PMID: 15736481.

28 Yuan J, Zhou J, Dong Z, Tandon S, Kuk D, Panageas KS, Wong P, Wu X, Naidoo J, Page DB, Wolchok JD and Hodi FS: Pretreatment serum VEGF is associated with clinical response and overall survival in advanced melanoma patients treated with ipilimumab. Cancer Immunol Res 2: 127-132, 2014. PMID: 24778276. DOI: 10.1158/2326-6066.CIR-13-0163

29 Dai DL, Martinka M and Li G: Prognostic significance of activated Akt expression in melanoma: a clinicopathologic study of 292 cases. J Clin Oncol 23: 1473-1482, 2005. PMID: 15735123. DOI: $10.1200 /$ JCO.2005.07.168

30 Davies MA, Stemke-Hale K, Lin E, Tellez C, Deng W, Gopal YN, Woodman SE, Calderone TC, Ju Z, Lazar AJ, Prieto VG, Aldape K, Mills GB and Gershenwald JE: Integrated molecular and clinical analysis of AKT activation in metastatic melanoma. Clin Cancer Res 15: 7538-7546, 2009. PMID: 19996208. DOI: 10.1158/1078-0432.CCR-09-1985

31 Pópulo H, Soares P, Faustino A, Rocha AS, Silva P, Azevedo F and Lopes JM: mTOR pathway activation in cutaneous melanoma is associated with poorer prognosis characteristics. Pigment Cell Melanoma Res 24: 254-257, 2011. PMID: 21029395. DOI: 10.1111/j.1755-148X.2010.00796.x

32 Corcoran RB, Rothenberg SM, Hata AN, Faber AC, Piris A, Nazarian RM, Brown RD, Godfrey JT, Winokur D, Walsh J, Mino-Kenudson M, Maheswaran S, Settleman J, Wargo JA, Flaherty KT, Haber DA and Engelman JA: TORC1 suppression predicts responsiveness to RAF and MEK inhibition in BRAFmutant melanoma. Sci Transl Med 5, 2013. PMID: 23903755. DOI: 10.1126/scitranslmed .3005753
Received April 19, 2019

Revised May 13, 2019

Accepted May 14, 2019 